\title{
Defining Pantomime for Language Evolution Research
}

\author{
Przemysław Żywiczyński ${ }^{1} \cdot$ Sławomir Wacewicz $^{1}$ (D) Marta Sibierska $^{1}$
}

Published online: 27 August 2016

(c) The Author(s) 2016. This article is published with open access at Springerlink.com

\begin{abstract}
Although pantomimic scenarios recur in the most important historical as well as current accounts of language origins, a serious problem is the lack of a commonly accepted definition of "pantomime". We scrutinise several areas of study, from theatre studies to semiotics to primatology, pointing to the differences in use that may give rise to misunderstandings, and working towards a set of definitional criteria of "pantomime" specifically useful for language evolution research. We arrive at a definition of pantomime as a communication mode that is mimetic; non-conventional and motivated; multimodal (primarily visual); improvised; using the whole body rather than exclusively manual; holistic; communicatively complex and self-sufficient; semantically complex; displaced, openended and universal. So conceived, "pantomime" is a near synonym of "bodily-mimetic communication" as envisaged by Donald and Zlatev. On a wider plane, our work may help organise some of the terminology and discussion in language evolution, e.g. by drawing a clear distinction between gestural and pantomimic scenarios or by specifying the relation between pantomimic and multimodal scenarios.
\end{abstract}

Keywords Pantomime - Mime - Gesture - Mimesis · Multimodality $\cdot$ Language origin $\cdot$ Language evolution

Sławomir Wacewicz

wacewicz@umk.pl

1 Department of English, Center for Language Evolution Studies CLES, Nicolaus Copernicus University, ul. Władysława Bojarskiego 1, 87-100 Toruń, Poland

\section{Introduction}

The rise of interest in the so-called pantomimic scenarios of language origins is evident in the works of several of the most influential scholars in this field, including Arbib (2005, 2008, 2009, 2012), Tomasello (2008), or the mimesis theorists Donald $(1991,2001)$ and Zlatev (2008) (cf. Cartmill and Goldin-Meadow 2012; McNeill 2013, for opposing views). The capacity of pantomime to represent and communicate relatively complex content without relying on pre-established meaning conventions, together with its apparent naturalness and universality, makes pantomime particularly noteworthy in the context of language evolution research. However, the proper classification and evaluation of the "pantomimic" models of language origins depend as much on their fit with available multidisciplinary evidence (cf. Wacewicz and Żywiczyński 2015), as on proper definitional groundwork. The underlying problem here is that the very notion of pantomime has not so far been analysed in great theoretical and empirical detail, and is used across a range of disciplines in ways that are considerably diverse and more intuitive than systematic. That this is so is even testified by researchers directly concerned with pantomime, such as McNeill, who at one point acknowledges "the lack of definition of pantomime" (2005: 6). What is required is a systematic and nuanced definition of pantomime and a better understanding of the mechanisms underlying its acquisition and cognitive processing.

Here, we aim at achieving this first, terminologicalconceptual, goal. We take a look at how the notion of pantomime functions across a variety of fields, from theatre studies or semiotics to primatology - to highlight the similarities but especially the areas of possible 
misunderstanding. Then, we arrive at a notion of pantomime that grows out of bodily mimesis (Donald 1991; Zlatev 2014), and can be defined as communication mode that is mimetic (volitional and representational); non-conventional and motivated; multimodal but primarily visual; improvised; using the whole body and the surrounding space rather than exclusively manual and stationary; holistic and non-segmental; communicatively complex and self-sufficient; semantically complex; displaced, openended and universal. Interestingly, the processing of pantomime so defined requires advanced cognitive capacities (e.g. triadic mimesis or perspective taking), a key feature that we do not have the resources to treat fully here.

\section{2 "Pantomime" Across the Disciplines}

\subsection{Theatre Studies}

"Pantomime" is most often translated as "an imitator of all/everything". The word has its roots in the theatrical tradition, and specifically originates from the Latin (ultimately Greek) panto-, meaning "all”, and mimos, ${ }^{1}$ referring to a "nonspeaking" performer who took on all the roles in a play and acted them out relying on masks, props and rhythmic movement. ${ }^{2}$ In Antiquity, this was synonymous with the performance of "a dancer" (cf. Slater 1994), who illustrated the tragic myths. Calling for great athletic ability, it resembled sports more than arts: it involved boxing and wrestling moves, high jumping, or somersaults (wasting the performers' energy, cf. Barba 1995: 15). Later on, this athletic repertoire of pantomimi was widened, as pantomime became an increasingly comical form, relying on mannerisms and exaggerations, which required the utmost precision of facial expression and gesturing (cf. Slater 1994).

In theatre studies, pantomime has thus been conceived of as a form of acting with the body; however, ideologically, it is not "a theatre where the actor does not speak, [it] is theatre where the actor's body does speak" (Lecoq in Peacock 2007: 217). In this sense, pantomime is a means of expression rather than a given-conventionalised-theatrical form. Lecoq, one of the most influential mime theorists and teachers, offered something of a prescriptive definition of thus understood pantomime, which, in his opinion, should be based on "corporal impression" and involve only "primal vocal sounds", being a "silent

\footnotetext{
${ }^{1}$ It is this latter part of the word, mimos (imitator, imitating), that carries the core meaning. This is reflected in the fact that the words "pantomime" and its shortened form, "mime", have very often been used interchangeably in theatre studies.

2 See e.g.: "pantomimus" in Encyclopaedia Britannica, http://www. britannica.com/art/pantomimus.
}

portrayal of real-life physical activity" (Lecoq in Peacock 2007: 217). Such an approach seems close to Decroux's corporeal mime or Stanislavsky's form of physical theatre, in which the movements of the performer should arise "genuinely" or "organically" in the course of improvisation (cf. Fleshman 2012: 206, Toporkov 2004: 159). It is worth noting that in these contexts, pantomime-though understood primarily as a dramatic form-is most often defined simply as "communicating through the use of gesture and movement rather than words", relying on "the visual and tactile channels of expression" (Peterson Royce 1992: 191).

Pantomime, understood as a form of a performance, has in general acquired a status of popular entertainment, ${ }^{3}$ and thus the term has been used rather reluctantly by theatre practitioners such as Stanislavsky or Grotowski, even though it is very close to what they called "physical actions" (cf. Spatz 2015: 139). The popular character of pantomime is also reflected in the interest that anthropological, ethnological and folklore studies take in the subject. In Bauman's edited volume on folk and popular entertainment forms, "mime" is listed alongside gossip, folktale, oral poetry, and ritual (1992). It is also present in almost every intra-cultural analysis of folklore of a given group or place: from Asia (e.g. Goonatilleka 1970, Lopez 2006), to Africa (Kerr 2005), to the Americas (Brunvand 1968). In Africa, for instance, mime has often been a way of combining the pre-colonial indigenous heritage of particular regions, usually in the form of original ritualistic dance, with parodying the colonial culture (Kerr 1995: 59-60). One of the most interesting forms is "militaristic mime". Kerr describes the Beni dance, which can be read as a parody of an army parade: the dancers, dressed in semi-military outfits, march in columns and mirror the behaviour of the European colonisers, using props that stand for rifles or batons (1995: 60). Another example is the Chama dance, in which the participants imitate an indigenous Arab sword combat, using sticks as props (1995: 60). These forms have a clear resemblance to the performances of the pantomimi of Ancient Greece and Rome: in one way or another they refer to fighting and require the military precision of movement.

Interestingly, "pantomime" also has an alternative etymology: for example, according to Broadbent (1901), it means "an imitator of Pan", the Ancient deity associated with Arcadia, and thus nature. In this sense, "pantomime" denotes "imitating nature", and connotes mimicry. This

\footnotetext{
${ }^{3}$ From the Roman pantomime, Italian commedia dell-arte, slapstick and silent movies, to street mimes - it has been associated with the so called low or popular art. A telling example is the present-day association of "pantomime" (or, in short, "panto") in British or more broadly Anglophone cultures with a particular genre of musical comedy, staged especially in the Christmas season.
} 
corresponds to the definitions of "expressive" pantomime that were provided in the twentieth century, stressing the connection of pantomime and imitation inherent in human nature. Lecoq, for instance, draws an analogy between miming and yawning, both of which are uncontrollable and catching (2006: 1-3). He seeks the roots of the drive to imitate and mimic also in the instances of copying gestures, postures, or behaviours of others that we are interacting with socially (2006: 3 ). The connection of mime and mimicry has also been observed by Broadbent; he uses the term [mimicry] in his History of Pantomime of 1901, where he writes that it is "the Pantomime of Nature, [...] which each and every one of us possesses in greater or lesser degrees, and as much as we do the Dramatic instinct" (1901: 14). By that, Broadbent points to the universal nature of pantomime, but also voices the intuition that the inclination towards using pantomime is inborn in humans; he refers, for instance, to Bernardin de St. Pierre, who observed that "[pantomime] was the first language of man; it is known to all nations; and is so natural and so expressive that the children of white parents learn it rapidly when they see it used by the negroes" (de St. Pierre 1788 in Broadbent 1901: 15). Still, Lecoq rightly states that mime is not synonymous with mimicry: it is not just mere imitation, but a way of "grasping the real", communicating something about it (cf. Lecoq 2006: 3). Another theatre theorist, Lust, calls pantomime "a language of gestures" (2003: 19; also-Lecoq 2006: 6), and she intuitively seeks its origins in pre-speech and its function in aiding verbal communication: "Before the human voice developed, gestures served not only to communicate but to aid in the development of vocal sounds. Later they were incorporated in the first forms of written language of, for example, the Egyptians, the Aztecs, and in the pictographic writings of the Hebrews" (2003: 20).

\subsection{Gesture Studies}

In terms of kinesiological or gestural research, the greatest effort to characterise pantomime was made by McNeill, who included it in his influential classification of gestural behaviours designated as Kendon's continuum (renamed as the "gesture continuum", at Kendon's request; cf. McNeill 2013):

Gesticulation-Language-like Gestures-PantomimeEmblems-Sign Languages (1992).

There are a number of parameters that dictate the organisation of the gesture continuum (or later, gesture continua, cf. 2005), but it is the relation of a particular gestural type to speech that is crucial to McNeill's classificatory enterprise: as explained above, gesticulations are defined by their close relation to spoken language, emblems are characterised by its optional presence, while pantomime and sign language are necessarily produced in the absence of speech (2005).

McNeill is not verbose about the details of pantomimic communication-“"[p]antomime is difficult to define, but generally it means a significant gesture without speech, a dumb show" (2005: 2). The examples given, e.g. twirling the finger around in a circle to stand for a vortex (2005), make it similar to gesticulations in the sense that pantomimes are non-conventional and spontaneous communicative movements (in contrast to emblems and signs). McNeill concedes that pantomimes are often elaborate, complex and potentially sequentially structured (2008, 2013), and that they must holistically refer to events, although he does not press this last point-it is principally the obligatory absence of speech and/or relation to linguistic utterances (as in the case of "language-slotted gestures", 2005) that distinguishes pantomime from gesticulation. $^{4}$

Importantly for our purposes, McNeill is a vehement opponent of the view that language evolved from pantomime, advanced by Arbib (e.g. 2012) or Tomasello (2008). For him, the defining feature of linguistic communication and indeed the evolutionary continuity in language evolution is the co-expressiveness of gesture and speech $(1992,2005,2013)$. Using the notion of "growth point", i.e. the psychological predicate of an utterance (loosely derived from Vygotsky 1962; McNeill 1992), he strives to show that linguistic communication rests on the scaffolding of gestures and lexemes, working together in meaning-making. This leads him to embracing the view of language as an essentially multimodal, or rather $b i$-modal, form of communication.

No researcher in gesture studies has devoted so much attention to pantomime as McNeill. Kendon (2004: 160) lists pantomime as one of three basic techniques of representation in gesturing; pantomime is the same as enactment, and is distinct from modelling and depiction in being action-oriented, i.e. tracing a pattern of action. Along similar lines, Sandler (2009) distinguishes pantomime from iconic signs or gestures: she proposes that iconic gestures refer symbolically by highlighting a salient feature of an object (e.g. the hands re-create the oval shape to indicate an egg), while pantomimes are action-oriented and consist in

\footnotetext{
4 "There are several distinguishing marks of PANTOMIME compared to gesticulation. One is that gesticulation integrates with speech; it is an aspect of speaking itself. PANTOMIME separates from speech. There is no co-construction, no co-expressiveness; timing is different (if there is speech at all), and no dual semiotic modes. PANTOMIME, if it relates to speaking at all, does so, as Susan Duncan points out, as a 'gap filler'-appearing where speech does not, the 'language-slotted' position of the Gesture Continuum, for example completing a sentence ("the parents were OK but the kids were [PANTOMIME of knocking things over]')" (McNeill 2016: $111)$.
} 
producing a mimetic replica of an action pattern (in the egg example, the hands can mime the action of breaking an egg and throwing away the shell). Streeck refers to mime as a form of performance related to "bodily quotations" or "enactments" that we engage in during social interactions in order to indicate our emotional states (2002: 591). Poggi (2007), in addition to their action-oriented iconicity, underscores the creative and novel aspect of pantomimes: they are absent from the mental "gestural lexicon" and need to be created on the spot. Pantomime is quite symptomatically absent from Ekman and Friesen's influential typology of non-verbal behaviours (1969).

Finally, an influential paradigm initiated by GoldinMeadow are laboratory studies on natural word-order in nonlinguistic representation of events; there, "elicited pantomime" is sometimes used to refer to enacting simple transitive events, such as a man pushing a box (e.g. Hall et al. 2013). Importantly, all the major studies in this line of research rely on a seated design that keeps the participants stationary and prevents them from using their whole bodies (Goldin-Meadow et al. 2008; Meir et al. 2010; Gibson et al. 2013; Hall et al. 2013); in effect, the type of communication studied there is impromptu manual communication (see below "silent gesture").

To sum up, the term "pantomime" in gesture studies functions in a rather broad variety of ways, motivated both by formal classifications of nonverbal behaviours and by the colloquial use of the word. McNeill's usage probably comes closest to being a stable technical term, but even leading authorities on gesture frequently move back and forth between the more technical and more intuitive meanings of "pantomime".

\subsection{Semiotics}

Several lines of research underscore pantomime's rich potential for carrying meanings. Thus, in traditional semiotics, pantomime is understood as a "nonverbal text" endowed with its own "grammar" (see e.g. De Marinis describing Decroux's changes in "his new grammar of physical mime", 1993: 125). In terms of the semiotics of performance in particular, pantomime is placed alongside gestures and movements, often in the company of dance or music (e.g. De Marinis 1993: 72, 79, 178). Much attention has also been given to the reading of the meaning of props used in pantomime (e.g. Wyles 2008, focusing on a semiotic-specifically, symbolic - analysis of costumes). This is different from the perspective of Experimental Semiotics, a subfield closely related to language evolution research, where pantomime has been used with an intuitive meaning closely overlapping "silent gesture" (e.g. Fay et al. 2013; 2014; Schouwstra 2012; Schouwstra and de Swart 2014)—i.e. silent, iconic depictions of individual concepts by means of one's hands and arms. Just like "elicited pantomime", silent gesture is visual, nonconventional, manual, segmental and simple. As such it has been associated with sign languages as a potential starting point in the evolution of this communication system (see e.g.: Roberts et al. 2015).

\subsection{Therapeutical Aspects}

Spontaneous miming not restricted by any syntax is often prescribed in teaching dyslexic people. Teachers' guides instruct, for instance, to: "mime something in the manner of the word (e.g. <run quickly>) and have the children find the right adverb", "have them [the children] mime the activity in the manner of the adverb while the other children guess the activity and the adverb" (Borwick 1999: 51), "[m]ime a particular nursery rhyme or incident and encourage the children to guess the mime. They can then choose something to mime in return" (Augur 1994: 153), or simply use mime in order to express ideas and emotions (Eadon 2005). Similarly, communicative body movements are used in psychotherapy; e.g. Dynamic Play Therapy combines body movements and narration in sessions for children with Hyperactivity or Attention Deficit Disorder (e.g. Harvey 2010); there are also techniques in Gestalt Therapy consisting in the exaggeration of communicative body movements to increase body awareness, which are used in a variety of affective disorders (e.g. Oaklander 1994).

In this sense, pantomime is synonymous with spontaneous gesturing, including both manual representations as well as whole-body enacting, aimed at conveying meanings that are usually conveyed through words. Its aim in therapy is either to provoke a guessing game that can aid the acquisition of new verbal semiotic resources, or to substitute verbal communication. Such an intuition underlies, for instance, the use of Makaton, the multimodal system of signs based mostly on iconicity that are either gestured or presented graphically. Some of the signs include combined gestures-icons, such as a hand's downward-upward movement, imitating picking something up, and putting it into one's mouth to designate the verb "to eat". Gesture combinations often accompany and aid speech in individuals with autism, cognitive and physical disabilities, or Down syndrome, whose verbal communication is restricted for a variety of reasons (see e.g. Grove and Walker 1990). Autistic people in particular rely in communication on mime signing or the so called kinaesthetic language. Mime signs mostly involve hand movements based on imitation, in the absence of speech, and, unlike sign language, they are not conventionalised. Kinaesthetic signing, in turn, often incorporates the whole of the body and aids translating words. For instance, the signing of the lexeme "jump" is accompanied by the performance of an actual jump (Bogdashina 2005: 232). 
The robustness of pantomime as a surrogate form of communication is testified to by the universality of pantomimic charades, found across times and cultures (Bellew 2011). Recently, this form of entertainment has received growing attention from various specialists, such as educators (Hidayati 2016) or therapists (Kaduson and Schaefer 2010), while the communicative potential of pantomime has been put to test in a variety of controlled assignment tasks, which can be as complex as designing a software system (Pavlov and Yatsenko 2005).

\subsection{Narratology}

Pantomime has been recognised not only as a means of communication, but also specifically as a medium for narrating. Abbott defines narrative and narrativity as "the representation of an event or a series of events", where "representation" is understood as any medium or modality, from a verbal and written work of literature, through an anecdote told in a casual conversation, to stories narrated not with words but with, for instance, gestures (2008: 13). In this sense, for narratologists, inspired by the intuitions of Jameson, Lyotard, or Barthes, a narrative is, next to language, a universal and uniquely human ability (Abbott 2008: 1-2).

Accordingly, pantomime seems to fit into the category of "a narrative medium". Peterson Royce states that it "is characterized by the features of narrative, time and space, an impulse and weight" (1992: 191). Although the intuition seems correct, in narratology gesturing and pantomime are most often seen as language-dependent media. In other words, they are viewed as suitable only for a limited repertoire of event sequences or stories, as they resolve in real time, from one gesture or movement to another, one by one-they cannot be re-arranged into flashbacks or flashforwards, nor represent anachronisms that are an indispensable part of narrating (cf. Genette 1980, 2002; Ryan 2012). Also, it has been claimed that pantomime can be successful in conveying a story only insofar as it relies on verbal aid: a (recognisable) title, libretto or programme (Ryan 2012). Indeed, in some pantomimic forms a libretto has been a part of the performance (in Antiquity sung by a chorus, now printed), but this seems true only in some cases of Western culture; with respect to the indigenous pantomime performances mentioned above, there is no evidence of incorporating any verbal aid.

\subsection{Primatology}

Of particular relevance to language origins research is the status of pantomime in our primate-especially apecousins. For a bona fide pantomimic model of language origins to stand, pantomime must be uniquely human, or at least human pantomime must be qualitatively different from any ape manifestations (cf. Tomasello 2008; Arbib 2012). In this context, it is interesting that despite a considerable body of research into primate gesture, only isolated systematic reports exist of iconicity in nonhuman apes (Tanner and Byrne 1996)—although motivational factors rather than cognitive limitations may be the reason (Genty and Zuberbühler 2015). This leads sceptics to suspect that the iconicity is only apparent and derives from "simpler" associative processes developing over ontogeny rather than from the cognitive processing of the iconic aspect of the signal (see Perlman et al. 2014 for review).

Consequently, the equally scarce available reports of pantomime in apes tend to assume a "leaner" rather than richer understanding of this notion: communicatively "demonstrating a particular action, usually to get a partner to perform that action or to request an associated object" (Perlman et al 2014: 230). Examples mostly come from enculturated apes, with the exception of a "form of pantomime" that is "potentially iconic" and consists in communication via demonstrating a sexual action, found in wild female bonobos (Douglas and Moscovice 2015). Worth noting is that reports of iconic gestures or pantomimes in non-human apes often demonstrate the multimodal character of such actions (viz. tactile gestures in Tanner and Byrne 1996) and the involvement of the whole body, e.g. in hip shimmies described by Douglas and Moscovice or hip shaking performed by bonobo mothers to invite the offspring for a ventral carry (Rossano 2013).

An intriguing exception are two works on forest-living orangutans by Russon and Andrews (2010, 2011). These reports adhere to an impressively rich understanding of pantomime: "gesture in which meaning is acted out; in humans, it can be as simple as twirling a finger to indicate a vortex or as complex as telling the Ramayana. It can be representational, symbolic, narrative in form and fictional [...] It can communicate meaning with sentential structure..." (Russon and Andrews 2010). The instances of pantomime identified in orangutans are described as productive, compositional, systematic, and triadically communicative (Russon and Andrews 2011) as well as multimodal, reenactive of past events, and communicatively versatile rather than tied to a particular function (Russon and Andrews 2010). However, no other nonanecdotal reports exist of ape behaviour meeting or approximating such "richer" criteria for pantomime. In sum, Zuberbühler's (2013: 136) conclusion appears to be largely accurate: "[in apes], pantomiming is conspicuously absent, apart from isolated anecdotes".

\subsection{Neuroscience}

Research on pantomime has been a long-standing element of neuroscience, with pantomimes being e.g. standard 
diagnostic tools for apraxia (Heilman et al. 1982; De Renzi et al. 1982; De Renzi and Faglioni 1999). However, following the tradition traced back to Hughlings Jackson (1893), pantomime has come to be understood in a very specific, and indeed very limited sense, qua imitating a simple action, i.e. execution of the relevant motor sequence in the absence of its instrumental goal, and in the absence of its object for transitive actions. Most typically, this action is pretend tool use, such as the use of (an imaginary) hammer or paintbrush, and can be glossed by a single verb or at most a simple verb phrase. It is based on a diagnostically important assumption that tool miming is related to motor programmes that are independent of any environmental features and are only conditioned by the characteristics of the mimed objects and activities; this is taken to contrast with gestures representing intransitive actions (e.g. hitchhiking), which are more dependent on social-cultural information and hence on the lexicon (for discussion see Bartolo et al. 2003). Standard studies in this paradigm focus on both the performance of pantomimes (e.g. Dumont et al. 1999), as defined above, and their comprehension (e.g. Rothi et al. 1985). Authors such as Feyereisen (1999) distinguish pantomime from imitation: pantomimes are elicited by verbal commands and imitation is elicited by the participant observing someone else execute the action.

With the expansion of neuroscience and advancement of scanning and imaging techniques in the 1990s, research on pantomime began to cover more and more thematic areas, some of them of great interest to language evolution, such as activation patterns induced by observing pantomime and signs of a sign language (Emmorey et al. 2010). However, this change of interest has not yet borne on a use of the term "pantomime" that is accepted in neuroscience.

\section{Towards Defining Pantomime}

The various uses of the term "pantomime" listed above appear to form a broad "family resemblance" category: it has no clear common core, consisting instead of several recurring prototypical features alongside rather important differences. For example, such differences are evident in the comparison between theatrical approaches with their focus on traditional, semi-conventionalised ways of pantomimic expression; and neuroscientific accounts, which view pantomime in diagnostic terms; or the gesturological perspective, which to a large degree has come to be dominated by McNeill and his research agenda. Below, we distil some of the most central features of pantomime, but delimit them in a way specifically geared to the goals of language evolution research.

\subsection{Mimetic}

Pantomime is volitional and representational, in that it relies on intentionally producing bodily forms that represent (stand for something other than themselves) in an imitative way. So conceived, pantomime is at the heart of what Donald (1991) and Zlatev (2008) have influentially called mimetic communication. Accordingly, it involves "the invention of intentional representation": body movements are devised to stand for an event either by a performance of a duplicate of what originally happened (mimicry sensu Donald) or by a less literal, more selective re-enactment (imitation sensu Donald). Hence, although pantomime incorporates both mimicry and imitation, it is the representational, i.e. mimetic, dimension that constitutes its defining feature. Donald envisages mimesis as a cognitive adaptation that allowed the hominin mind to break away from the here-and-now characteristic of the non-human apes (i.e. the episodic culture). In this sense, mimesis, although inherently able to perform the communicative function, is really a cognitive adaptation and can be used outside the communicative context (e.g. as the rehearsal of a tool-making process).

On the ontological ground, this makes pantomime organism-external rather than organism-internal (on the analogy of the Chomskyan E-language vs. I-language). It is a mode of communication that follows from mimesis as the underlying cognitive ability, and fulfils the definitional criteria set for mimesis by Donald, such as intentionality, i.e. the objective of pantomime is to represent an event; referentiality, i.e. pantomime stands for something distinct from itself; or autocueing, pantomimes are self-generated, that is their production is volitionally controlled (Donald 1991). ${ }^{5}$

More specifically, pantomime relies on what Zlatev (e.g. 2008, Zlatev et al. 2005) calls triadic mimesis ("speakeraddressee-referent"). Accordingly, pantomime involves sharing representations between self and other, construed as the producer and the intended receiver; as already noted, pantomime is volitional and representational in that it depends on the differentiation of the signifier (bodily act)—from the signified (a represented event); finally, it has a triadic nature comprised of a pantomime, its addressee but also its meaning, i.e. the intention that a body movement be recognised as intentionally communicative. The last point, following Zlatev, should be interpreted as testament of pantomime having the Gricean character (2008). We must note here that from the language-origins

\footnotetext{
5 While on the present account 'pantomime' is almost synonymous to 'mimetic communication', 'pantomime' is historically much more established in language origins scenarios, and is much more intuitively meaningful than 'mimesis' to persons from outside the field.
} 
vantage point, such communication presupposes honest signalling_a "platform of trust" (Dor et al. 2014)_as a precondition.

In one way or another, many of the features discussed in points 3.2 through 3.8 result from unpacking the term "mimetic communication".

\subsection{Motivated and Non-conventional}

Pantomime relies on motivated semiosis, i.e. it exploits iconicity in the sense of some inherent resemblance between the form of expression and the intended meaning. Admittedly, both iconicity and, more generally, similarity are notoriously problematic explanatory principles (cf. Goodman 1972; Nöth 2008; Żywiczyński 2010); however, some kind of "natural" form-to-meaning link necessarily follows from the absence of pre-established consensual links. ${ }^{6}$ This absence, that is the lack of (semiotic) conventionality, is critically important from the language origins perspective, where the central problem is accounting for the emergence of conventions.

Following on from Müller's work on how practical action changes into gesture (e.g. 2014), we note that pantomimes employ action patterns reduced with respect to those found in the actions that pantomimes represent (as in the pantomime of eating an apple set against the very action of eating an apple), with the salient features of an event becoming abstracted and schematised. Such a mechanism of change from practical action to pantomimic representation widely opens the door to conventionalization; however, pantomimic signification is accomplished by virtue of the iconic relationship between the signifier and the signified, and not the fact that this relationship is shared by a community of users. Hence, pantomime is nonconventional and non-normative; for instance, while it is true that some pantomimic re-enactments are better than others in terms of e.g. communicative success, it is impossible to make a pantomimic "mistake" in the same way that one makes a linguistic mistake. This is also why we prefer to call pantomime a "mode" or "means" rather than a "system" of communication.

The requirement of semiotically non-conventionalised meaning has several interesting consequences. Firstly, from the present perspective the various forms of expression that fall back upon conventions could not count as pantomime (e.g. the game of charades-see Arbib 2012: 177, 217). Furthermore, we could expect pantomime to have some level of universality as opposed to culture-specificity, so that forms of expression whose comprehension or

\footnotetext{
$\overline{6}$ Although motivation and conventionality are not necessarily mutually exclusive, the lack of one implies the other by logical necessity.
}

interpretation largely differs between cultures (or is highly sensitive to one's cultural background) might thereby fail to qualify as pantomime in the relevant sense.

\subsection{Improvised}

Without the normative aspect that comes with conventions, pantomimes are spontaneous and impromptu. Arbib (2012, 2013) repeatedly makes this point in his origins scenario by stressing that first pantomimes are "ad hoc", "artless" or "naïve". Such pantomimes are necessarily creative, as their one-off nature implies that signs must be coined on the spot and interpreted on the spot, rather than simply retrieved from memory; so the invention of signals also takes place online, in the real-time dynamics of the communicative situation (cf. Poggi 2007: 192-194). Of course, this relatively unstandardised character of pantomimes leads to considerable disadvantages in terms of time, cognitive cost, and communication efficiency; and if some forms do get replicated-lower replication fidelity. As stated by Hutto (2008: 269), "It is not easy to communicate by means of pantomime [...]. To be sure it is a hit and miss affair: definitely more miss than hit". Other authors comment on this drawback in a similar spirit (Arbib 2012: 219; Corballis 2015: 91), and a generally agreed conclusion is that such problems would be a powerful incentive for the conventionalization of pantomimic forms.

\subsection{Multimodal (But Primarily Visual)}

Contrary to some of the traditional uses (cf. Slater 1994 in theatre studies above), where pantomime is silent by definition, in the language origins context there is no need to postulate unimodal-visual pantomime. In fact, on the present account pantomime is fully compatible with multimodality, and pantomimic performance may subsume concurrent sound production ("primal vocal sounds", typically of emotional character), provided the vocalisation is non-conventional and has limited referential potential. This again accords with Donald's conception of mimetic communication, where the main transmission channel is visuomotor (i.e. "motor" on the production side and "visual" on the reception side) comprised of "all forms of hand and limb movement, postural attitudes, and locomotor movements" (1991: 77). The dominance of the visuomotor channel in pantomime is dictated by its iconic potential, superior to the vocal-auditory channel in the context of iconically bootstrapping a communication system (see e.g. Fay et al. 2013, 2014). However, mimetic communication is not at all constrained to this one channel but makes active and frequent use of other modalities and semiotic resources- "facial expression and other modes of emotional expression, such as a variety of calls and cries, and 
strictly prosodic aspects of voice modulation would also have fitted into a purely mimetic culture" (Donald 1991: 78).

This leads to the idea of vocal pantomime and more generally of pantomime as a multimodal mode of expression (e.g. including also tactile gestures or non-vocal bodily generated sounds, such as clapping, thumping and the like), in which the transmission of information depends on the joint effect of the different semiotic resources (including vocal imitation, music and dance) working together for complementary or even synergistic effects. In our view, multimodality reveals an important cognitive mechanism involved in the production of pantomimes. On the most fundamental ground, as argued by Zlatev with reference to bodily mimesis (2008: 219), pantomime requires "a crossmodal mapping between exteroception (i.e. perception of the environment, normally dominated by vision) and proprioception (perception of one's own body, normally through kinaesthetic sense)". The effective use of various sensory modalities in one communicative (i.e. pantomimic) act calls for a cognitive infrastructure that operates supramodally, being able to construe and execute a coherent event re-enactment through the use different channels of expression. Note that many existing "pantomimic" scenarios have precisely such a multimodal nature, from the early speculations of Condillac (1746) to contemporary scenarios such as the one proposed by Mithen (2005).

\subsection{Using the Whole Body and the Surrounding Space}

Pantomime represents a communication mode characterised by the absence of language and relying on movements of the whole body - mainly manual gestures, body movements, facial expressions or voluntarily enacted mannerisms. Accordingly, the communicative potential of pantomime depends on the intentional use of integrated movement of multiple body parts to convey meaning. It is further magnified by the use of the peripersonal and public space when the mime may convey ideas by reference to elements of the immediate environment, such as landmarks, including previous positions of own body. Contrast this with exclusively manual gestures, such as emblems or iconic imitations of simple instrumental action (see: Neuroscience), which on the present account would not count as "pantomimes" in and of themselves. Of course, movements of the hand and arm do have a rather critical part to play in pantomime, but only to the extent that they are implicated in the holistic generation of meaning, rather than being used in isolation from other body movements.

Another reason for defining pantomime as involving the whole body is dictated by the comparative context. As already noted, the cases of iconic gestures and so-called pantomimes attested in non-human apes tend to involve movements of the whole body rather than isolated manual actions (Tanner and Byrne 1996; Russon and Andrews 2010, 2011; Rossano 2013; Douglas and Moscovice 2015). An implication is that the stationary and almost exclusively manual silent gesture/elicited pantomime paradigm might not be telling us the whole story from the language origins perspective.

An interesting upshot is the resulting production effort. The relatively high energetic cost of producing pantomime leads to consequences that are noteworthy in the language evolution context. On the one hand, high energetic expenditure upholds signal honesty, which would make pantomime suitable for implementing costly rituals (Power 2009: 271). On the other hand, once the platform of trust is established in the community and the risk of deception is minimised, pressures on energy efficiency would apply. This is evidenced both in laboratory experiments (e.g. Roberts et al. 2015) and diachronic sign language studies (e.g. Fusellier-Souza 2006; Klima and Bellugi 1979; see also Kendon 2004: 309), which show that as signals become more conventionalised in successive generations of communicators, they also tend to simplify and involve gradually less production effort.

\subsection{Holistic}

The holistic nature of pantomime also pertains to its structure as a communicative act. Here, pantomime again differs from individual gestures (e.g. emblems), which have a clear onsettermination structure and correspond to clearly identifiable, discrete concepts. Such movements usually have glosses consisting of one lexeme and could in principle replace individual words in verbal messages or combine and recombine to form systematic, compositional messages (see McNeill's "language-slotted gestures" below). The above is also true of homesigns in deaf children, who "could easily (and effectively) convey information by producing continuous and unsegmentable movements in mime-like fashion [...] But the deaf children don't behave like mimes. They produce discrete gestures concatenated into sentences-their gestures resemble beads on a string rather than one continuous strand" (Goldin-Meadow 2005: 189-190). In contrast, pantomime refers to whole events or sequences of events in a holistic "continuous strand", with no self-apparent onsets and terminations in the stream of movement, which does not naturally decompose into easily isolable component parts. While it may be possible to single out segments as a matter of postfactum analysis, such segments would lack obvious discrete boundaries and may not be freely recombinable.

\subsection{Communicatively Complex and Self-Sufficient}

Consistently with their holistic nature, pantomimic acts are "the size of" propositions or utterances rather than smaller 
component units; rather than being elements of a larger communicative whole, they express complete, self-contained communicative acts. This requirement is again a quite direct and fundamental consequence of the languageorigins context: since pantomime is a candidate for phylogenetically bootstrapping conventional communication, it cannot depend on the co-presence of conventional semiotic resources. This is why, again, isolated gestures (e.g. imitation of action) substituting for individual words or phrases in a sentence would not form self-standing pantomimes. The self-sufficiency requirement means that pantomime must be able not only to form self-contained communicative acts, but also be independent of any other help from verbal resources, i.e. it must be comprehensible in the absence of any verbally established context.

\subsection{Semantically Advanced: Displaced, Open-Ended and Semantically Universal}

Pantomimic communication is a semantically rich and sophisticated mode of expression. Firstly, it has displacement in the sense of Hockett (1960), i.e. the potential to refer to entities not present in the immediate spatiotemporal vicinity of the communicator. This ability to go beyond the "here and now" underlies the usefulness of pantomimic communication, which is thus capable of transmitting information of great social and ecological importance. For this reason, displacement is quite vital to the main pantomimic scenarios of language emergence (cf. Arbib 2012; Tomasello 2008). The property of displacement again puts pantomime in the position of a "bridge": although nonlinguistic, it possesses what many consider the most fundamental property of human as opposed to animal communication (e.g. Deacon 2011; Hurford 2011). In terms of Dor (2015), pantomime has the analog richness of experiential communication typical of non-linguistic systems, but shows some hallmarks of detached, skeletal schematisation typical of instructive (linguistic) communication (see also Perniss and Vigliocco 2014, on how iconicity could bootstrap displaced communication).

For similar reasons, important features of pantomime are open-endedness and semantic universality, i.e. the ability to convey a potentially unlimited set of messages bearing on all types of semantic domains. Following Stokoe and others, Arbib (2012: 219) sees the main power of pantomime in "the ability to create an open-ended set of complex messages exploiting the primates' open-ended manual dexterity". At this point we should note that the claim is not absolute, in the sense of presupposing full displacement, open-endedness and universality-the limitations of pantomime in conveying certain kinds of e.g. highly abstract content are an interesting empirical question (cf. Ryan 2012). However, a system of communication severely limited to the here and now, to a predefined range of signals, or to a fixed set of semantic domains would not qualify as a qualitatively novel stage in language evolution, and consequently would not substantiate a reference to a "pantomimic scenario".

\section{Defining Pantomime}

To reiterate, in view of the above definitional analysis, we take pantomime to be a non-verbal, mimetic and nonconventionalised means of communication, which is executed primarily in the visual channel by coordinated movements of the whole body, but which may incorporate other semiotic resources, most importantly non-linguistic vocalisations. Pantomimes are acts of improvised communication that holistically refer to a potentially unlimited repertoire of events, or sequences of events, displaced from the here and now. In doing so, pantomime does not depend on semiotic conventions.

Finally, it would be useful to briefly consider some examples of various forms of visual, bodily meaningmaking that by present criteria do not qualify as "pantomime”. It is revealing to notice that many contemporarily used communicative behaviours would be ruled out on the grounds of their conventionalisation. Examples are emblems and signs of a sign language, which-even if having detectable iconicity - are clearly conventional (also: isolated rather than holistic, and mostly manual-only). Such is also the case with Tic-tac signs (cf. Waterman 1999) and other similar domain-specific systems (also: isolated, not semantically universal, and mostly manual-only), and less obviously, activities such as charades (a point observed by Arbib 2012). There are also reasons to believe that most contemporary miming as a theatrical performance relies on conventional signals to an unexpectedly large degree.

The homesigns of deaf children not using a sign language, although clearly iconic and sometimes even called "pantomimic", are segmental rather than holistic (GoldinMeadow 2005). Co-speech gesturing might again contain identifiable iconicity, but is not self-contained in requiring obligatory co-presence of speech, and also there are limits as to its volitionality, representationality and semanticreferential potential. Language-slotted gestures, which McNeill (2013, but compare McNeill 1992) takes as instantiating "pantomimes", are likewise dependent on copresent speech. Imitation of instrumental actions, esp. tooluse, commonly called pantomimes in the neuroscientific literatures (see above), is isolated, mostly manual-only, communicatively simple and not self-sufficient.

Finally, silent gesture and elicited pantomime are particularly interesting types of communication, having recently risen to the status of important experimental 
paradigms in broadly construed language evolution studies (Fay et al. 2013, 2014; Goldin-Meadow et al. 2008). They are both non-linguistic, improvised acts of communication executed in the visual channel, which-taking the bulk of Donald's and Zlatev's criteria-should be classified as instances of mimetic communication. However, they are stationary and manual (not involving the movement of the entire body), communicatively simple and usually segmental rather than holistic. Their most pronounced difference from pantomime-the selective use of manual gestures as the means of expression-is the effect of the chosen experimental design. Like pantomime, silent gesture can be multi-, or rather, bi-modal with non-linguistic vocalisation accompanying gesturing, whereas elicited pantomime tends to be performed in the visuomotor channel only. However, with regard to the type of referents, pantomime as defined here is similar to "elicited pantomime", where the usual referents are transitive events, and differs from silent gesture, which mainly serves to express isolated lexical concepts. We could say that pantomime inhabits the very centre of the "mimetic communication" category, while silent gesture and elicited pantomime are its less prototypical members.

\section{Conclusion}

The central position that pantomime has come to occupy in language origins calls for careful definitional work, specifically geared towards the needs of language evolution research. After all, the key to classifying a particular position as advocating a pantomimic scenario, to a large extent, lies in the definition of pantomime. This is particularly important in an interdisciplinary field such as language evolution, and in the case of a notion such as pantomime, which serves a variety of research goals and intellectual sensibilities.

Language origins scenarios that aspire to the name of pantomimic cannot work with a "lean" definition but need a rich one, such that the emergence of pantomime could qualify as a truly novel and qualitatively different stage in language evolution. Our discussion of the various senses and uses of pantomime defends such a complex definition, which however does not defy the intuitive understanding of the notion and is at the same time regimented enough to afford meaningful comparisons. Needless to say, we do not consider our proposal as the definitive formulation of such a definition: we hope it is the first of many steps-discussions and arguments-which will eventually bring a definition encapsulating all the aspects of pantomime relevant to language evolution and in so doing bear on language evolution research itself. Finally, we see the mechanisms underlying the acquisition and cognitive processing of pantomime as a particularly important direction for further research.

Acknowledgments This research was funded by the Faculty of Languages, Nicolaus Copernicus University, research fund. We are grateful to Casey Lister, Konrad Juszczyk, Martin Edwardes, Jordan Zlatev and Daniel Dor for their valuable comments on an earlier draft of this paper. All remaining errors are our own.

\section{Compliance with Ethical Standards}

Conflict of interest The authors declare that they have no conflict of interest.

Open Access This article is distributed under the terms of the Creative Commons Attribution 4.0 International License (http://crea tivecommons.org/licenses/by/4.0/), which permits unrestricted use, distribution, and reproduction in any medium, provided you give appropriate credit to the original author(s) and the source, provide a link to the Creative Commons license, and indicate if changes were made.

\section{References}

Abbott HP (2008) The Cambridge introduction to narrative. Cambridge University Press, Cambridge

Arbib MA (2005) From monkey-like action recognition to human language: an evolutionary framework for neurolinguistics. Behav Brain Sci 28:105-167

Arbib MA (2008) From grasp to language: embodied concepts and the challenge of abstraction. J Physiol 102(1):4-20

Arbib MA (2009) Evolving the language-ready brain and the social mechanisms that support language. $\mathrm{J}$ Commun Disord 42(4):263-271

Arbib MA (2012) How the brain got language. Oxford University Press, Oxford

Arbib MA (2013) Complex imitation and the language-ready brain. Lang Cogn 5(2-3):273-312

Augur J (2003) [1994] Early help means a better future. In: Hales G (ed) Dyslexia matters. Whurr Publishers Ltd, London, pp $150-158$

Barba E (1995) The paper canoe. A guide to theatre anthropology. Routledge, London

Bartolo A et al (2003) Pantomimes are special gestures which rely on working memory. Brain Cogn 53:483-494

Bauman R (ed) (1992) Folklore, cultural performances, and popular entertainments. A communications-centered handbook. Oxford University Press, Oxford

Bellew F (2011) The art of amusing. Ulan Press, San Bernardino

Bogdashina O (2005) Communication issues in autism and asperger syndrome. Do we speak the same language? Jessica Kingsley Publishers, London

Borwick C (1999) Spoken language. In: Townend J, Turner M (eds) Dyslexia in practice: a guide for teachers. Springer, New York, pp 31-56

Broadbent RJ (1997) [1901] A history of pantomime. Arno Press, New York

Brunvand JH (1968) The study of American Folklore: an introduction. WW Norton, Chicago

Cartmill EA, Goldin-Meadow S (2012) Is pantomime a likely stage in language evolution? Evidence from human and primate gesture. The evolution of language: proceedings of the 9th international conference (EVOLANG9). World Scientific, New Jersey, p 418 
Corballis M (2015) The wandering mind: what the brain does when you're not looking. The University of Chicago Press, Chicago

De Marinis M (1993) The semiotics of performance. Indiana University Press, Bloomington

de Condillac ÉB (2001) [1746] An essay on the origin of human knowledge. Cambridge University Press, Cambridge

De Renzi E, Faglioni P (1999) Apraxia. In: Denes G, Pizzamiglio L (eds) Handbook of clinical and experimental neuropsychology. Psychology Press, Hove, pp 421-441

Deacon TW (2011) The symbol concept. In: Gibson KR, Tallerman $M$ (eds) The Oxford handbook of language evolution. Oxford University Press, Oxford, pp 393-405

Donald M (1991) Origins of the modern mind: three stages in the evolution of culture and cognition. Harvard University Press, Cambridge

Donald M (2001) A mind so rare. The evolution of human consciousness. Norton, New York

Dor D (2015) The instruction of imagination: language as a social communication technology. Oxford University Press, Oxford

Dor D, Knight C, Lewis J (2014) Introduction: a social perspective on how language began. In: Dor D et al (eds) The social origins of language. Oxford University Press, Oxford, pp 1-14

Douglas PH, Moscovice LR (2015) Pointing and pantomime in wild apes? Female bonobos use referential and iconic gestures to request genito-genital rubbing. Sci Rep. doi:10.1038/srep13999

Dumont C et al (1999) Selective impairment of transitive gestures: an unusual case of apraxia. Neurocase 5:447-458

Eadon H (2005) Dyslexia and drama. David Fulton Publishers Ltd, Abington

Ekman P, Friesen WV (1969) The repertoire of nonverbal behavior: categories, origins, usage, and coding. Semiotics 1:49-98

Emmorey K et al (2010) CNS activation and regional connectivity during pantomime observation: no engagement of the mirror neuron system for deaf signers. Neuroimage 49:994-1005

Fay $\mathrm{N}$ et al (2013) How to bootstrap a human communication system. Cogn Sci 37:1356-1367

Fay N et al (2014) Creating a communication system from scratch: gesture beats vocalization hands down. Front Psychol. doi:10. 3389/fpsyg.2014.00354

Feyereisen P (1999) Neuropsychology of communicative movements. In: Messing LS, Campbell R (eds) Gesture, speech, and sign. Oxford University Press, Oxford, pp 3-26

Fleshman R (2012) Etienne Decroux and the contemporary theatre: Decroux corporeal mime. In: Lust A (ed) Bringing the body to the stage and screen. Expressive movement for performers. The Scarecrow Press Inc., Lanham, pp 205-214

Fusellier-Souza I (2006) Emergence and development of signed languages: from a semiogenetic point of view. Sign Lang Stud $7(1): 30-56$

Genette G (1980) [1930] Narrative discourse. An essay in method. Cornell University Press, New York

Genette G (2002) Order, duration, and frequency. In: Richardson B (ed) Narrative dynamics: essays on time, plot, closure, and frames. The Ohio State University, Columbus, pp 25-34

Genty E, Zuberbühler K (2015) Iconic gesturing in bonobos. Commun Integr Biol. doi:10.4161/19420889.2014.992742

Gibson E et al (2013) A noisy-channel account of crosslinguistic word-order variation. Psychol Sci 24(7):1079-1088. doi:10. $1177 / 0956797612463705$

Goldin-Meadow S (2005) The resilience of language: what gesture creation in deaf children can tell us about how children learn language. Psychology Press, New York

Goldin-Meadow S et al (2008) The natural order of events: How speakers of different languages represent events nonverbally. Proc Natl Acad Sci. doi:10.1073/pnas.0710060105
Goodman N (1992) [1972] Seven strictures on similarity. In: Douglas M, Hull DL (eds) How classification works: Nelson Goodman among the social sciences. Edinburgh University Press, Edinburg, pp 13-22

Goonatilleka MH (1970) Mime, mask and satire in Kolam of Ceylon. Folklore 81(3):161-176. doi:10.1080/0015587X.1970.9716678

Grove N, Walker M (1990) The Makaton vocabulary: using manual signs and graphic symbols to develop interpersonal communication. Augment Altern Commun 6(1):15-28. doi:10.1080/ 07434619012331275284

Hall ML et al (2013) Cognitive constraints on constituent order: evidence from elicited pantomime. Cognition 129(1):1-17

Harvey S (2010) The scarf story. In: Kaduson H, Schaefer Ch (eds) 101 Favourite play therapy techniques. Rowman \& Littlefield Publishers Inc, New York, pp 46-49

Heilman KM et al (1982) Two forms of ideomotor apraxia. Neurology 32(4):342-346

Hidayati NR (2016) The use of charade game to teach vocabulary. J Engl Lang Teach 5(1). http://journal.unnes.ac.id/sju/index.php/ elt/article/view/9887/6327. Cited 19 May 2016

Hockett CF (1977) [1960] Logical considerations in the study of animal communication. In: The view from language: selected essays 1948-1974. The University of Georgia Press, Athens, pp 124-162

Hughlings JJ (1893) Words and other symbols in mentation. In: Taylor J (ed) (1958) Selected writings of John Hughlings Jackson: Vol 2. Evolution and dissolution of the nervous system, speech, various papers, addresses and lectures. Basic Books, New York, pp 205-212

Hurford JR (2011) The origins of meaning. In: Gibson KR, Tallerman $M$ (eds) The Oxford handbook of language evolution. Oxford University Press, Oxford, pp 370-381

Hutto D (2008) First communions: mimetic sharing without theory of mind. In: Zlatev $\mathbf{J}$ et al (eds) The shared mind: perspectives on intersubjectivity. John Benjamins Publishing Company, Amsterdam, pp 245-276

Kaduson H, Schaefer Ch (eds) (2010) 101 Favourite play therapy techniques. Rowman \& Littlefield Publishers Inc, New York

Kendon A (2004) Gesture: visible action as utterance. Cambridge University Press, Cambridge

Kerr D (1995) African popular theatre: from pre-colonial times to the present day. James Currey Ltd, Oxford

Kerr D (2005) Theater: African popular theater. In: Peek PM, Yankah K (eds) African Folklore: an encyclopaedia. Routledge, New York, pp 932-937

Klima E, Bellugi U (1979) The signs of language. Harvard University Press, Cambridge

Lecoq J (2006) Theatre of movement and gesture. Routledge, London

Lopez ML (2006) A handbook of Philippine Folklore. The University of the Philippines Press, Diliman

Lust A (2003) From the greek mimes to Marcel Marceau and beyond: mimes, actors, pierrots, and clowns: a chronicle of the many visages of mime in the theatre. Scarecrow Press, Lanham

McNeill D (1992) What gestures reveal about thought. The University of Chicago Press, Chicago

McNeill D (2005) Introduction. In: McNeill D (ed) Language and gesture. Cambridge University Press, Cambridge, pp 1-12

McNeill D (2008) Gesture and thought. University of Chicago Press, Chicago

McNeill D (2013) The co-evolution of gesture and speech, and downstream consequences. In: Müller $\mathrm{C}$ et al (eds) Bodylanguage-communication, vol 1 . Walter de Gruyter, Berlin, pp 480-512

McNeill D (2016) Why we gesture: the surprising role of hand movements in communication. Cambridge University Press, Cambridge 
Meir I et al (2010) The interaction of animacy and word order in human languages: a study of strategies in a novel communication task. In: Smith A et al (eds) Eight evolution of language conference. World Scientific, Utrecht, pp 455-456

Mithen S (2005) The singing neanderthals: the origins of music, language, mind and body. Weidenfeld and Nicholson, London

Müller C (2014) Gestural modes of representation as techniques of depiction. In: Müller $\mathrm{C}$ et al (eds) Body-language-communication, vol 1 and 2. Walter de Gruyter, Berlin, pp 1687-1702

Nöth W (2008) Semiotic foundations of natural linguistics and diagrammatic iconicity. In: Willems K, De Cuypere L (eds) Naturalness and iconicity in language. John Benjamins, Amsterdam, pp 73-100

Oaklander V (1994) Gestalt play therapy. In: Schaefer ChE, O'Connor KJ (eds) Handbook of play therapy, advances and innovations. Wiley, New York, pp 143-156

Pavlov LV, Yatsenko A (2005) The babel experiment: an advanced pantomime-based training in OOA and OOD with UML. In: Proceedings of SIGCSE, St. Louis, pp 231-235

Peacock DK (2007) Changing performance. Culture and performance in the British Theatre since 1945. Peter Lang, Oxford

Perlman M et al (2014) Iconicity and ape gesture. In: Evolution of language: proceedings of the 10th international conference (EVOLANG10). World Scientific, New Jersey, pp 228-235

Perniss P, Vigliocco G (2014) The bridge of iconicity: from a world of experience to the experience of language. Philos Trans R Soc B 369(1651):20130300

Peterson Royce A (1992) Mime. In: Bauman R (ed) Folklore, cultural performances, and popular entertainments. A communicationscentered handbook. Oxford University Press, Oxford, pp 191-195

Poggi I (2007) Mind, hands, face and body: a goal and belief view of multimodal communication. Weidler, Berlin

Power C (2009) Sexual selection models for the emergence of symbolic communication: why they should be reversed. In: Botha R, Knight C (eds) The cradle of language. Oxford University Press, Oxford, pp 257-280

Renzi De et al (1982) Modality-specific and supramodal mechanisms of apraxia. Brain 105:301-312

Roberts G, Lewandowski J, Galantucci B (2015) How communication changes when we cannot mime the world: experimental evidence for the effect of iconicity on combinatoriality. Cognition 141:52-66. doi:10.1016/j.cognition.2015.04.001

Rossano F (2013) Sequence organization and timing of bonobo mother-infant interactions. Interact Stud Soc Behav Commun Biol Artif Syst 14(2):160-189

Rothi LJ et al (1985) Pantomime comprehension and ideomotor apraxia. J Neurol Neurosurg Psychiatry 48(3):207-210
Russon A, Andrews K (2010) Orangutan pantomime: elaborating the message. Biol Lett. doi:10.1098/rsbl.2010.0564

Russon A, Andrews K (2011) Pantomime in great apes: evidence and implications. Commun Integr Biol 4(3):315-317

Ryan ML (2012) Narration in various media. In: Hühn P, Meister J Ch, Pier J, Schmid W (eds) The living handbook of narratology. http://www.lhn.uni-hamburg.de/article/narration-various-media. Cited 1 May 2016

Sandler W (2009) Symbiotic symbolization by hand and mouth in sign language. Semiotica 174(1/4):241-275

Schouwstra M (2012) Semantic structures, communicative strategies and the emergence of language. LOT Dissertation Series 312. LOT, Utrecht

Schouwstra M, de Swart H (2014) The semantic origins of word order. Cognition 131:431-436

Slater WJ (1994) Pantomimes. Didaskalia 1(2). http://www.didaska lia.net/issues/vol1no2/wslater.html\#. Cited 1 May 2016

Spatz B (2015) What a body can do. Technique as knowledge. Practice as research. Routledge, London

Streeck J (2002) Grammars, words, and embodied meanings: on the use and evolution of so and like. J Commun 52(3):581-596

Tanner JE, Byrne RW (1996) Representation of action through iconic gesture in a captive lowland gorilla. Curr Anthropol 37(1):162-173

Tomasello M (2008) Origins of human communication. MIT Press, Cambridge

Toporkov V (2004) Stanislavski in rehearsal. Routledge, New York

Vygotsky L (1962) Thought and language. MIT Press, Cambridge

Wacewicz S, Żywiczyński P (2015) From the narrow to the broad. Multiple perspectives on language evolution. Theoria et Historia Scientiarum 11:5-18

Waterman J (1999) The Punter's friend. Queen Anne Press, Harpenden

Wyles R (2008) The symbolism of costume in ancient pantomime. In: Hall E, Wyles R (eds) New directions in ancient pantomime. Oxford University Press, Oxford, pp 61-86

Zlatev J (2008) From proto-mimesis to language: evidence from primatology and social neuroscience. J Physiol 102(1):137-151

Zlatev J (2014) Image schemas, mimetic schemas, and children's gestures. Cogn Semiot 7(1):3-29

Zlatev J, Persson T, Gärdenfors P (2005) Bodily mimesis as "the missing link" in human cognitive evolution. Lund University Cognitive Studies 121

Zuberbühler K (2013) Acquired mirroring and intentional communication in primates. Lang Cogn 5(2-3):133-143

Żywiczyński P (2010) Axiology of spoken interaction. An essay on the organisation of conversational behaviour. Wydawnictwo Naukowe UMK, Toruń 